\title{
Application and Research in the Drill Reliability Design Based on Fuzzy Theory
}

\author{
Dong Li \\ Xi'an Research Institute, China Coal Technology and Engineering Group Corp, Xi'an 710077, China
}

\begin{abstract}
In order to improve the reliability of the coal mine tunnel drilling rig in the absence relevant data at the primary design stage, the reliability fuzzy prediction model and the fuzzy comprehensive evaluation process was established based on the fuzzy theory in this paper. The reliability of hydraulic chuck was predicted by the reliability index of some key slips, further the whole drill reliability index was analyzed, it provided a new way for tunnel drill design. The experimental results showed that the theory of fuzzy reliability prediction could improve drill reliability and provide theoretical basis and technical support for design.
\end{abstract}

\section{Keywords-formatting; style; styling; insert}

\section{INTRODUCTION}

China is the biggest coal-producing country in the world, it is a multiple tectonic plate through multiple order tectonic movement patchwork of land, and the condition of geological structure is very complicated. There is large difference of coal resources distribution, poor geological conditions, especially the gas, water and other disasters threaten seriously. With the mining depth increasing, all kinds of the disasters threat has become increasingly worse ${ }^{[1]}$. Coal mine tunnel drilling is widely used in gas extraction, drain construction, geological exploration, it is the essential equipment of coal mine safety and efficient production. As the Fig.1 shows the system of coal mine tunnel drilling rig. If any part is the failure, the machine wills not work ${ }^{[2]}$. Because of the particularity environment, the drill rig was long stored under harsh conditions and repeated use, it puts forward higher requirements on the reliability of the drilling rig. Therefore, it is very necessary that proposed to analysis and study about the prediction, analysis and evaluation on the reliability of drilling rig.

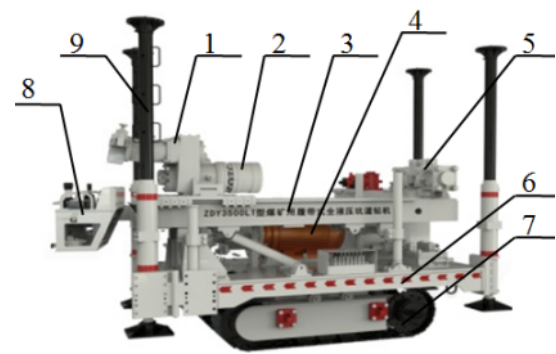

1-gyrator; 2-chuck; 3-feed device; 4-motor pumps; 5-holder; 6 - bodywork platform; 7 - track; 8 - console; 9 - solid device

FIGURE I. THE SYSTEM OF COAL MINE TUNNEL RIG
The core of reliability design is through the basic unit of the reliability index to estimate the system reliability index of reliability prediction and allocation, optimization and management, both applied to various engineering system of common theoretical foundation and calculation principle, and involves specific attributes and characteristics of each system ${ }^{[3]}$. It is considered that the influence of some factors such as functional, technical level, the influence of the working condition of environment, economy and so on, in addition to the geometric relationship and movement. There are the fuzziness in these factors that are difficult to determine with value, it leads to the ambiguity tunnel drill design. Fuzzy theory is widely used in the robot, the complex mechanical and electrical system, electric power, agriculture and other fields ${ }^{[4-7]}$, but in the coal mine equipment and the application of reliability prediction research is less. The reliability fuzzy prediction model and the fuzzy comprehensive evaluation process was established based on the fuzzy theory in this paper, it provided a new way for tunnel drill design.

\section{THEORY OF RELIABILITY FUZZY PREDICTION}

\section{A. The Establishment of the Fuzzy Evaluation Factor}

According to the characteristics and main performance index of the predicted equipment, many factors that will affect the certain properties of the equipment reliability was divided into $\mathrm{p}$ class, the factors set $U=\left(\begin{array}{llll}u_{1}, & u_{2}, & \ldots, & u_{n}\end{array}\right)$ was divided into p subsets: $U=(U 1, U 2, \ldots, U p)$.Among them, $U_{i}=(i=1,2, \ldots, p)$ was expressed the $i$ class, and meet: $U_{1} \cup U_{1} \cup \cdots \cup U_{1}=U$ and $U_{1} \cap U_{1}=\phi,(i \neq j)$.

\section{B. The Establishment of the Weight set}

Establishment of the weight set is fuzzy membership degree of each factor. Membership can reflect the factors status and role in comprehensive evaluation, it directly affects the final fuzzy evaluation values. Generally, coal mine hydraulic tunnel drilling rig as a complex system of mutual correlation operation, all aspects of the various parts and components should have the same importance, but in different circumstances, the demonstrated importance of various factors are different. The weight set $A=\left(\begin{array}{llll}a_{1}, & a_{2}, & \cdots, a_{p}\end{array}\right)$, meet $0<a_{i} \leq 1, \quad \sum_{i=1}^{p} a_{i}=1$. 


\section{The Establishment of the Fuzzy Relationship Matrix}

The factors of evaluation set for: $b_{i}=\left(b_{i 1}, b_{i 2}, \cdots, \quad b_{i m}\right),(i=1,2, \ldots, p)$. There is a relative membership degree for each judge, it can be seen as the standard values of each level are: $S=\left(S_{1}, S_{2}, \ldots, S_{m}\right)$. Each single factor evaluation is jointed, it can get a set of factors from the fuzzy relation matrix of the evaluation $\mathrm{Q}$, marked as:

$$
Q=\left(\begin{array}{c}
b_{1} \\
b_{2} \\
\vdots \\
b_{p}
\end{array}\right)=\left(\begin{array}{cccc}
b_{11} & b_{12} & \cdots & b_{1 m} \\
b_{21} & b_{22} & \cdots & b_{2 m} \\
\vdots & \vdots & \cdots & \\
b_{p 1} & b_{p 2} & \cdots & b_{p m}
\end{array}\right)_{i j}
$$

Among them, each line in the $Q b_{i}=\left(\begin{array}{llll}b_{i 1}, & b_{i 2}, & \ldots, & b_{i m}\end{array}\right)$, $(i=1,2, \ldots, p)$ are the evaluation results of the $i$ factor, the $b_{i j}$ is the $j$ membership degree for each $i$ judge, it reflects fuzzy relationship between the evaluation factors and expressed in membership degree. The establishment of the fuzzy evaluation matrix is used by expert evaluation method, which experts use experience on factors relative to its evaluation factors focused on the subjective evaluation. The $d$ shows the number of the participated evaluation experts in the $Q$, the $d_{i j}$ shows the number of the $j$ membership degree for each $i$ judge evaluation experts.

\section{The Establishment of the Evaluation Matrix and the Unit Score}

The relationship between the factor weight set $\mathrm{A}$ and judging matrix $Q$ was established, according to the principle of fuzzy transformation, available for fuzzy evaluation matrix model was: $B=A \circ Q=\left(B_{1}, B_{2}, \ldots, B_{S}\right)$, comprehensive evaluation score was: $C=B \circ S^{T}$. According to the characteristic of the index system of fuzzy operator, this is the lower index upper composite index evaluation vector at various levels or value calculation method. The determination of fuzzy operator is the determination of fuzzy synthesis methods. There are five common evaluation models. The first is $M(\wedge, \vee)$, its synthetic operation is $B_{j}=\stackrel{p}{\vee}\left(a_{i=1} \wedge b_{i j}\right),(j=1,2, \ldots, m)$. The second is $M(\bullet, \vee)$, its synthetic operation is $B_{j}=\underset{i=1}{\vee p}\left(a_{i} \bullet b_{i j}\right)$, $(j=1,2, \ldots, m)$. The third is $M(\wedge, \oplus)$, its synthetic operation is $B_{j}=\min \left[1, \sum_{i=1}^{p}\left(a_{i} \wedge b_{i j}\right)\right],(j=1,2, \ldots, m)$. The fourth is $M(\bullet, \oplus)$, its synthetic operation is $B_{j}=\min \left[1, \sum_{i=1}^{p}\left(a_{i} \bullet b_{i j}\right)\right]$, $(j=1,2, \cdots, m)$. The last one is $M(\bullet,+)$, its synthetic operation is $B_{j}=\sum_{i=1}^{p}\left(a_{i} \bullet b_{i j}\right),(j=1,2, \ldots, m), \sum_{i=1}^{n} a_{i}=1$.

\section{FUZZY PREDICTION OF SYSTEM RELIABILITY}

Reliability prediction is defined as the method of fuzzy reliability prediction to quantitatively estimate the future product at the design stage, based on the reliability of the components of the system.

If system parts can be used normally after repaired, it will be called its average trouble-free working time, marked as $M T B F, M T B F=\sum_{i=1}^{N} t_{i} / N, t_{i}$ shows the $i$ part trouble-free working time and $N$ shows the total failure times among them.

The longevity of the mechanical system parts obeys exponential distribution $f(t)=\lambda e^{-\lambda t}$, the unit failure rate $\lambda$ is made as constant, then $M T B F=1 / \lambda$. If the system is composed of each unit series, the units are usually assumed independent and equal working time, system reliability $\mathrm{R}$ is equal to the reliability of product each unit, $R=R_{1} R_{2} \cdots R_{n}=\prod_{i=1}^{n} R_{i}, R_{i}=e^{-\lambda_{i}}$, $\lambda_{i}=\lambda C_{i}$, the $\lambda_{i}$ shows the $i$ unit failure rate, the $\lambda$ shows the failure rate of a known unit, the $C_{i}$ shows the $i$ unit the integrated score coefficient.

\section{ApplicAtion In the Design of TUNNEl DRILl CHUCK}

Hydraulic chuck is an independent important part of drilling rig, due to its worse working conditions and frequent action, it was able to sustain the largest axial load and the maximum torque. The drilling efficiency and quality were affected by chuck's performance. The Fig.2 shows the chuck of coal mine tunnel rig, the main failure shows slip abrasion, and the reliability of the slip can be use to predict the reliability of the chuck.

The factors effecting the reliability of chuck slip were determined according to the actual working condition and experts in the set $U=\{u 1, u 2, u 3, u 4, u 5\}=\{$ design level, importance degree, complexity, environmental conditions, working time $\}$, and the weight set $A=(0.15$, $0.25,0.100 .15,0.35)$, the relative membership degree $\mathrm{S}=(0.15,0.25,0.100 .15,0.20)$. The Tab.1 shows the definition of evaluation grades $b i=\left\{b_{i 1}, b_{i 2}, b_{i 3}, b_{i 4}\right.$, $\left.b_{i 5}\right\}, \quad(i=1,2, \cdots, 5)$.

The fuzzy relationship matrix was listed according to the factor evaluation scale:

$$
Q=\left(\begin{array}{ccccc}
0.2 & 0.3 & 0.3 & 0.1 & 0.1 \\
0.5 & 0.4 & 0.1 & 0 & 0 \\
0.4 & 0.3 & 0.2 & 0.1 & 0 \\
0 & 0 & 0.1 & 0.3 & 0.6 \\
0.7 & 0.2 & 0.1 & 0 & 0
\end{array}\right)
$$

Fuzzy operator select weighted average model. It may retain all useful information, high credibility, the synthesis 
operation for $B_{j}=\sum_{i=1}^{p}\left(a_{i} \bullet b_{i j}\right), \quad(j=1,2, \cdots, m), \quad \sum_{i=1}^{n} a_{i}=1$. Therefore, $B=A \circ Q=\left(\begin{array}{lllll}0.440 & 0.245 & 0.140 & 0.070 & 0.105\end{array}\right)$.

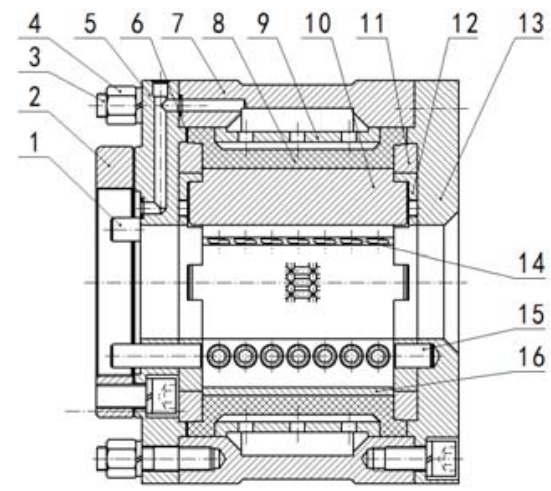

1 - long pin; 2 - the plate; 3-double end studs; 4-nut; 5 - rear end cover;6 - pad ring; 7 - chuck body; 8 - rubber tube; 9 - support ring; 10 - slip; 11 - pressure ring; 12 - the twisting plate; 13 - front end cover; 14 - spring; 15 - short pin; 16 - plate

FIGURE II. THE CHUCK OF COAL MINE TUNNEL DIG

TABLE I. DEFINITION OF EVALUATION GRADES

\begin{tabular}{|l|l|l|l|l|l|}
\hline $\begin{array}{l}\text { Evaluation } \\
\text { Factors }\end{array}$ & \multicolumn{4}{|l}{ Grades } \\
\hline Design level & highest & higher & general & lower & lowest \\
\hline $\begin{array}{l}\text { Importance } \\
\text { Degree }\end{array}$ & much & more & most & less & little \\
\hline Complexity & little & less & much & more & most \\
\hline $\begin{array}{l}\text { Environment } \\
\text { al Conditions }\end{array}$ & good & better & best & bad & worse \\
\hline $\begin{array}{l}\text { Working } \\
\text { Time }\end{array}$ & short & shorter & long & longer & longest \\
\hline
\end{tabular}

Comprehensive evaluation score was: $C=B \circ S^{T}=0.234$

Coal mine tunnel drill chuck is composed of 16 major parts, and slip is used as its main easily damaged parts. It was calculated by the standard of slip parts failure rate and the comprehensive evaluation score, The results showed that sixteen parts failure rate in slip for reference were $5 \%$, the obtained gripper reliability was $82.93 \%$, $R=\prod_{i=1}^{16} R_{i}=\prod_{i=1}^{16} e^{-\lambda_{i}}=e^{-\sum_{i=1}^{16} \lambda_{i}}=e^{-16 \lambda C}=0.8293$.

\section{CONCLUSION}

According to the slip reliability index to estimate the reliability of the chuck index and the holder of reliability index to estimate the reliability of the machine, which provided a method for reliability prediction of the machine. At the system design stage lack of reliability data, using fuzzy forecast method is the key to set up to predict the connection between the unit and the known unit reliability, though only in ideal situation provided approximate estimate values, it can provide for the designer with the reliability of the effective information feedback, which has important reference value and provides a new idea for improving the drilling machine reliability.

\section{REFERENCES}

[1] Qiang WU. Problems and Prospects of Prevention and Control Technology of Mine Water and Reclamation in China. Journal of China Coal Society, 2014, 39(5): 795-805. In Chinese.

[2] Dong LI. Research on Maintainability Design of Hydraulic System for Coal Mine Tunnel Rig. Machine Tool \& Hydraulics, 2012, 40(3):170174. In Chinese.

[3] SiJin TIAN. Reliability Analysis of the Accident Probability and Frequency Calculation on Ergonomic System. Journal of Shanghai Institute of Technology, 2002, 2(4): 231-234. In Chinese.

[4] ShengJun CHEN. Study on Reliability Prediction Theory of Robot System. Robot, 2003, 25(7): 648-651. In Chinese.

[5] JianYing GUO, YongQuan SUN, ChunYu Yu. Theory and Method for Complex Mechanical and Electrical System Reliability Prediction. Journal of Mechanical Engineering, 2014, 50(14): 1-13. In Chinese.

[6] Hua YAN, Jie WU, ZhiQiang MA. Applications of Fuzzy Set Theory to Short-term Load Forecasting in Power System. Automation of Electric Power Systems, 2000, 6: 34-38. In Chinese.

[7] ManZhi TAN, Jie ChEN, FangMing XU. Spatial Prediction of Soil Heavy Metal Pollution Based on Fuzzy Theory. Acta Pedologica Sinica, 2014, 50(4): 1-13. In Chinese. 